\title{
Power Management Strategy by Enhancing the Mission Profile Configuration of Solar-Powered Aircraft
}

\author{
Parvathy Rajendran, Kah Wee Lim, and Kuan Theng Ong \\ School of Aerospace Engineering, Universiti Sains Malaysia, Engineering Campus, 14300 Nibong Tebal, Pulau Pinang, Malaysia \\ Correspondence should be addressed to Parvathy Rajendran; paru80_2000@hotmail.com
}

Received 4 November 2015; Revised 6 May 2016; Accepted 24 May 2016

Academic Editor: William W. Liou

Copyright ( 2016 Parvathy Rajendran et al. This is an open access article distributed under the Creative Commons Attribution License, which permits unrestricted use, distribution, and reproduction in any medium, provided the original work is properly cited.

Solar energy offers solar-powered unmanned aerial vehicle (UAV) the possibility of unlimited endurance. Some researchers have developed techniques to achieve perpetual flight by maximizing the power from the sun and by flying in accordance with its azimuth angles. However, flying in a path that follows the sun consumes more energy to sustain level flight. This study optimizes the overall power ratio by adopting the mission profile configuration of optimal solar energy exploitation. Extensive simulation is conducted to optimize and restructure the mission profile phases of UAV and to determine the optimal phase definition of the start, ascent, and descent periods, thereby maximizing the energy from the sun. In addition, a vertical cylindrical flight trajectory instead of maximizing the solar inclination angle has been adopted. This approach improves the net power ratio by $30.84 \%$ compared with other techniques. As a result, the battery weight may be massively reduced by $75.23 \%$. In conclusion, the proposed mission profile configuration with the optimal power ratio of the trajectory of the path planning effectively prolongs UAV operation.

\section{Introduction}

The current high endurance of UAV has been achieved by harvesting solar energy through photovoltaic cells [1-8]. The sun has been used as an inexhaustible source for the longendurance flight of UAV. However, solar panels can generate power only at certain times during the day. Hence, rechargeable batteries have been introduced as secondary power storage to store the excess energy that can be discharged during nighttime. Moreover, an appropriate trajectory control in gravitational gliding during nighttime has been considered an efficient method to enhance flight endurance. The energy stored as gravitational potential energy and gained in height is used by the UAV to glide at night without any electrical power supply $[3,9]$.

Some studies focus on UAV kinematics to enhance the performance of UAV. The bank angle of UAV is crucial for determining the incidence angle of the sun on the solar panel for optimal solar harvesting [10]. Other studies specify the heading angle and UAV attitude in flight maneuvers to compensate for the sun's relative position for maximum solar harvesting $[11,12]$. However, a flight path that "follows" the sun consumes additional energy.

Another path planning method, also known as energy management strategy (EMS) $[13,14]$, has been studied to manage an efficient path for UAV using all the above methods. The idea of EMS is to divide the flight trajectory into three phases and utilize the solar energy that can be stored partly as gravitational potential energy during daytime. UAV harvests solar energy during daytime and gains gravitational potential energy that is employed during the gliding stage.

The maximum sun inclination angle was determined by changing the UAV's heading angle, which resulted in higher energy consumption because of the need for establishing a path following the sun [12]. Saghar and Mehran [2] also suggested that the potential energy harvested during the climb phase can be saved for further use. Another study focused on the trajectory along the surface of a vertical circular cylinder with optimized ascending and descending periods to increase the wing's sun exposure [15]. However, its mission phases are yet to be optimized for better UAV endurance. 
There are also researches done that suggested that the power net gain can be maximized by referring to local solar spectral density from weather forecasts [16]. Nevertheless, flexible path planning was performed [17], where the path was selected based on the cost function. This plan enables solarpowered unmanned aerial vehicle (UAV) to conduct perpetual flights and even allows for planetary missions by using discretization to solve the approximations of optimal paths $[18-20]$.

Moreover, several works have ignored the upper and lower boundary limits of the battery's state of charge (SOC). These limits must be between 0.25 and 0.90 to prolong the battery's shelf life and fully utilize the battery pack installed in any UAV [14]. Furthermore, battery weight is approximately $30 \%$ of the overall weight of UAV $[1,8]$. Thus, reducing battery weight may also significantly reduce the overall weight of UAV.

Framed by these contexts, this study adopts the optimal mission phase configuration of solar-powered UAV to optimize energy harvesting with minimal power consumption, to maximize the overall net power ratio, and to reduce the battery weight. Here, an alternative approach is presented by adopting an optimal mission phase's configuration with vertical cylindrical trajectory to increase the ratio of power gained to power consumed.

The sun elevation angle has also been considered to optimize the phases of the UAV flight, which include cruise, ascent, and descent, and to maximize the energy from the sun. This optimization has also minimized the battery capacity required and enables the reduction of the battery weight to maintain perpetual flight. As a result, the overall power consumption is reduced as the overall weight of the UAV is reduced. In addition, the proposed strategy enables the UAV to fly at a low altitude trajectory to avoid the highly volatile wind region at higher altitudes [21].

\section{Mathematical Model}

2.1. Aircraft Trajectory. In this section, a recently developed aircraft trajectory model is elaborated [12]. In an inertial $(x, y, z)$ Cartesian coordinate system shown in Figure 1, the $3 \mathrm{D}$ vectors of $x(t), y(t)$, and $z(t)$ are expressed as (1)-(3), respectively:

$$
\begin{aligned}
& x(t)=r \cos (\varphi(t)), \\
& y(t)=r \sin (\varphi(t)), \\
& z(t)=z(t-1)+\dot{z}(t) .
\end{aligned}
$$

The aircraft is assumed to fly in still air with a constrained radius $r$. The aircraft pitching angle $\theta$ and heading angle $\varphi$ change according to the boundary condition of the optimal flight velocity $V_{\text {Power }_{\min }}$ and ceiling altitude $z$. The vectors velocity $V$ and acceleration $a$ are expressed in terms of a moving coordinate system attached to the aircraft. The tangential, normal, and vertical components of velocity are expressed as $V_{t}, V_{n}$, and $V_{z}$ using (4)-(6), respectively, whereas the

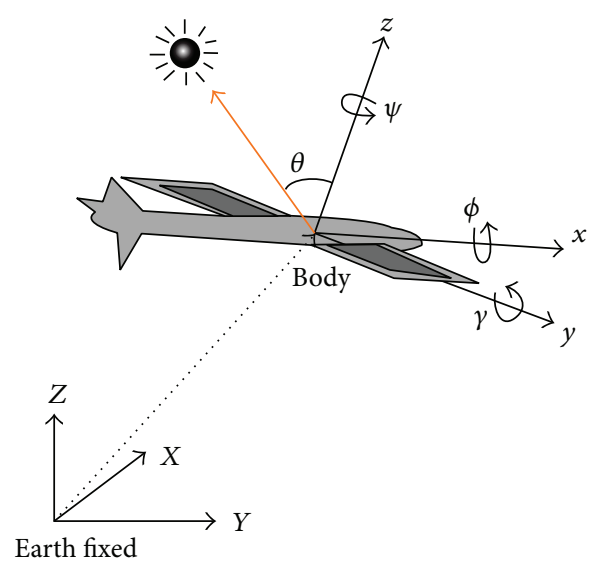

Figure 1: Cartesian coordinate system [3].

corresponding acceleration equations are expressed as $a_{t}, a_{n}$, and $a_{z}$ in (7)-(9), respectively [18]:

$$
\begin{aligned}
& V_{t}(t)=r \dot{\varphi}(t), \\
& V_{n}(t)=0, \\
& V_{z}(t)=\dot{z}(t), \\
& a_{t}(t)=r \ddot{\varphi}(t), \\
& a_{n}(t)=r \dot{\varphi} 2(t), \\
& a_{z}(t)=\ddot{z}(t) .
\end{aligned}
$$

The flight path angle $\gamma(t)$ between the aircraft tangential and ascending velocity vectors [18] is measured as described in

$$
\gamma(t)=\tan ^{-1} \frac{V_{z}(t)}{V_{t}(t)}=\tan ^{-1} \frac{\dot{z}(t)}{r \dot{\varphi}(t)} .
$$

The optimum flight velocity is the velocity at the minimum required power $V_{\text {Power }_{\min }}$ (see (11)) to sustain a level flight [18]. The rate of change of the heading angle $\dot{\varphi}(t)$ (see (12)) is determined using this optimum flight velocity and the radius $r$ constraint of the trajectory [18]:

$$
\begin{aligned}
V_{\text {Power }_{\min }} & =\sqrt[4]{\frac{4 K(m g)^{2}}{3 C_{D_{0}} \rho_{\infty}^{2} S^{2}}}, \\
\dot{\varphi}(t) & =\frac{V_{\text {Power }_{\min }}}{r} .
\end{aligned}
$$

2.2. Aircraft Force and Equation of Motion. According to the Cartesian coordinate system, the equations of motions are derived from three axes, as shown in [18]

$$
\begin{aligned}
m r \ddot{\varphi} & =(T-D) \cos \gamma+L_{t}, \\
m r \dot{\varphi}^{2} & =L_{n}, \\
m \ddot{z} & =-m g+(T-D) \sin \gamma+L_{z},
\end{aligned}
$$


where drag $D$ and thrust $T$ force are measured along the velocity vector. These equations can be rearranged to compute the corresponding lift required by the tangential, normal, and vertical components of velocity and the total lift expressed in (14)-(17), respectively [18]:

$$
\begin{aligned}
L_{t} & =m r \ddot{\varphi}-(T-D) \cos \gamma, \\
L_{n} & =m r \dot{\varphi}^{2}, \\
L_{z} & =m \ddot{z}+m g-(T-D) \sin \gamma, \\
L & =\sqrt{L_{t}^{2}+L_{n}^{2}+L_{z}^{2}} .
\end{aligned}
$$

2.3. Aircraft Aerodynamics. The thrust vector is assumed to be aligned with the free-stream velocity, which satisfies the small angle of attack. $K$ is the aerodynamic coefficient (see (18)), $\rho_{\infty}$ is the atmospheric density, $V$ is the magnitude of velocity, $e$ is the Oswald efficiency factor, AR is the aspect ratio of the wing, and $\alpha$ is the angle of attack [18]. Hence, the lift coefficient $C_{L}$, drag coefficient $C_{D}$, and drag force $D$ can be derived and computed from (19)-(21), respectively:

$$
\begin{aligned}
K & =\frac{1}{\pi e \mathrm{AR}}, \\
C_{L} & =\frac{2 L}{\rho_{\infty} V^{2} S}=C_{l_{0}}+C_{l_{\alpha}}, \\
C_{D} & =C_{D_{0}}+K C_{L}^{2}, \\
D & =\frac{1}{2} \rho_{\infty} V^{2} S C_{D} .
\end{aligned}
$$

2.4. Energy Modeling. This section explains the energy gained and lost during a flight, where the energy required, gained, and lost during the flight is discussed in terms of charged and discharged battery capacity. The standard electric propulsion model is used to derive the power to sustain a flight, as shown in (22) [12]. Kinetic and potential energy are conserved and excluded from the energy model. Thus, the output energy $E_{\text {out }}$ from the battery storage is shown in (23) [12]:

$$
\begin{aligned}
& P_{\text {out }}= \begin{cases}\frac{T V}{\eta_{\text {prop }}}, & T \geq 0 \\
0, & T \leq 0,\end{cases} \\
& E_{\text {out }}=\int_{t_{0}}^{t_{f}} P_{\text {out }} d t .
\end{aligned}
$$

In addition, the UAV is assumed to be fully mounted with solar cells on the wings, and the effective area of solar energy collection is assumed to be $S$, which is the area of the wing $[11,12]$. The location and date are chosen to obtain the solar spectral density Psd and solar elevation angles, se. The flight path angle and angle of attack are integrated as pitch angle $\theta$ [12]:

$$
\begin{aligned}
& \theta=\gamma+\alpha \\
& \cos (i)=\cos (\mathrm{se}) \sin (\theta) \sin (\mathrm{az}-\varphi)+\sin (\mathrm{se}) \cos (\theta) .
\end{aligned}
$$

The solar inclination $i$ is calculated using (25) [12]. The solar power generated is a piecewise function (see (26)) [12]. This power depends on the conversion efficiency, which, in turn, depends on the temperature and incident solar radiation [22-24]. In addition, the conversion efficiency also depends on the spectral and angular selectivity of the PV cells [25]. Besides, the efficiency of this system may be improved when integrated with maximum power-point tracker and optimizing its speed control [26-28]. The power input $P_{\text {in }}$ is considered zero when the inclination angle $i$ is negative, where $\eta_{\text {sol }}$ is the solar cell efficiency [24] and Psd is the solar spectral density [12]. Thus, the total energy input $E_{\text {in }}$ is the integral component of the generated solar power, as shown in (27) [12]:

$$
\begin{aligned}
& P_{\text {in }}= \begin{cases}\eta_{\text {sol }} \text { Psd } S \cos (i), & \cos (i) \geq 0 \\
0, & \cos (i)<0,\end{cases} \\
& E_{\text {in }}=\int_{t_{0}}^{t_{f}} P_{\text {in }} d t .
\end{aligned}
$$

The battery power output and capacity are based on a lithium-sulfur rechargeable battery [11], with a battery pack mass and power output that can be expressed as (28)-(29), respectively [11]. The state of charge, SOC, represents the ratio of the current charge capacity to the maximum charge capacity of the battery pack. The upper and lower SOC boundaries are recommended for the health and safety of the battery life, as given by $(30)[1,7]$. The SOC change rate SOCC can be expressed as (31) [11]:

$$
\begin{aligned}
m_{\mathrm{bat}} & =\frac{\left(Q V_{\mathrm{oc}}\right)}{\eta_{E_{b}}}, \\
P_{\mathrm{bat}} & =-V_{\mathrm{oc}} Q \mathrm{SOCC}-R(Q S \dot{O} \mathrm{C})^{2}, \\
0.25 \mathrm{C} \leq \mathrm{SOC} \leq 0.90 \mathrm{C}, & \\
\mathrm{SOCC} & =-\frac{V_{\mathrm{oc}}-\sqrt{V_{\mathrm{oc}}^{2}-4 P_{\mathrm{bat}} R}}{2 Q R(3600)} .
\end{aligned}
$$

The performance of the battery is affected by temperature. However, the working temperature is in the range of low altitude temperature. Battery discharge and charge temperature's lowest boundary is $-20^{\circ} \mathrm{C}$. This temperature allows the battery to operate efficiently until the altitude of $5500 \mathrm{~m}$, which is lower than the assumed ceiling altitude.

\section{Mission Profile Strategy}

A solar-powered aircraft mission profile is generally divided into three main phases: cruise, ascent, and descent. During the cruise flight, the UAV employs energy from the battery to sustain a level cruise flight. The ascent flight begins when the UAV starts to gain its gravitational potential energy and stores the excess energy fed into the battery. The descent flight of the UAV occurs when the aircraft starts to glide without using any power source. The power required to control the surface 


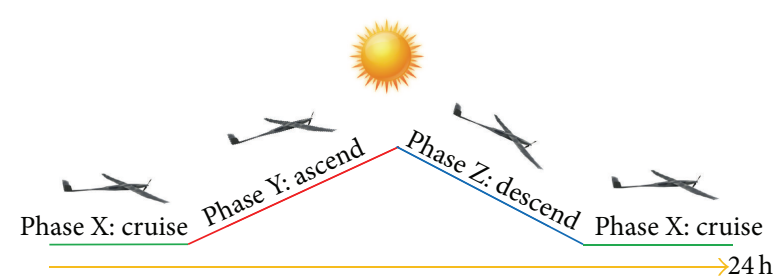

Figure 2: Mission phase.

operation may also be disregarded. The UAV continues to cruise when it reaches an initial altitude of $100 \mathrm{~m}$. The mission phases are illustrated in Figure 2.

3.1. Phase X: Cruise Flight. At cruise, the aircraft begins to discharge the battery with the minimum power required to sustain a level flight. In this simulation, the altitude is set to clearance of $100 \mathrm{~m}$ to avoid geographical obstruction. The UAV is also set to fly at the minimum power to maximize the UAV's endurance in this phase. In addition, the final SOC of this phase is not lower than the battery SOC boundary of $0.25 \mathrm{C}$, as recommended by previous researchers $[1,7,8]$.

3.2. Phase Y: Ascent Flight. In the ascent phase, the UAV climbs steadily with the available excess power at a speed that can be calculated using (32). The UAV is constrained by a radius $r$. Thus, the bank angle, angle of attack, and flight path angle of the UAV vary accordingly. In this phase, the flight is estimated to harvest more energy than the consumed energy. Hence the net energy is positive:

$$
\dot{z}=\frac{\text { ceiling }}{\text { Daylight time }} \text {. }
$$

3.3. Phase Z: Descent Flight. In this phase, the UAV starts to employ the gravitational potential energy collected during the ascent phase for unpowered glide. Thus, it is assumed that the UAV does not consume any power to sustain its level flight during the descent phase. The minimal descent rate $V_{\text {sink }}$ is desirable for maximizing the UAV's endurance. Thus, the UAV must maintain its flight at the maximum $C_{L}^{3 / 2} / C_{D}$ to obtain a minimal sink rate [18]. The ratio $C_{L}^{3 / 2} / C_{D}$ and $V_{\text {sink }_{\text {min }}}$ are defined in (33) and (34), respectively [18]:

$$
\begin{aligned}
\frac{C_{L}^{3 / 2}}{C_{D}} & =\frac{1}{4}\left(\frac{3}{K C_{D_{0}}}\right)^{3 / 4}, \\
V_{\text {sink }_{\min }} & =\frac{1}{\left(C_{L}^{3 / 2} / C_{D}\right)_{\max }} \sqrt{\frac{2}{\rho_{\infty}} \frac{m g}{S}} .
\end{aligned}
$$

3.4. Proposed Power Management Strategy. The proposed power management strategy for the mission profile configuration aims to optimize the time management of the mission phase with respect to the elevation angles of the sun to maximize solar energy. Flying according to the sun's
TABLE 1: UAV model data.

\begin{tabular}{lc}
\hline Parameter & Value \\
\hline$C_{D_{0}}$ & 0.011 \\
$\eta_{\text {prop }}$ & 0.7 \\
$\eta_{\text {sol }}$ & 0.22 \\
$b$ & $0.711 \mathrm{~m}$ \\
$S$ & $0.1566 \mathrm{~m}^{2}$ \\
$\eta_{E_{b}}$ & 0.992 \\
Aircraft weight & $4.201 \mathrm{~N}$ \\
$m_{\text {bat }}$ & $0.317 \mathrm{~N}$ \\
$Q$ & $7.65 \mathrm{Ah}$ \\
$V_{\text {oc }}$ & $12.6 \mathrm{~V}$ \\
$R$ & $0.0125 \Omega$ \\
\hline
\end{tabular}

inclination angle does not significantly affect energy efficiency because this consumes more power than the amount of energy generated from the sun.

The adaptation of a vertical cylindrical trajectory minimizes the battery mass. Hence an optimal net power is obtained during the flight. Moreover, this study adopts an additional element by defining the optimal time of mission transition in relation to the elevation angle of the sun to improve the overall power ratio gained. This structure of the mission phases, in which the start-end-time of the ascent, cruise, and descent phases of a flight are defined, leads to better energy savings and therefore improves aircraft endurance.

The three phases are looped in the time function for 24 hours, starting from 0:15 hours until 00:00 hours at the interval of 15 minutes to obtain the optimal time to start each phase. Although the elevation angle and intensity of the sun differ at each hour, the UAV still flies according to the planned path without following the sun's angle. Thus, no extra energy is used in the performance of the sun-following maneuver, but changes in solar intensity over time are utilized to improve the final performance.

\section{Results and Discussion}

The power ratio and SOC obtained at each simulation are analyzed to determine the optimal mission profile configuration. The result shows that the power management strategy improves the net power ratio of a solar-powered UAV (i.e., aircraft specification given in Table 1), as discussed in the following subsection. Section 4.2 illustrates the simulation scheme to analyze the feasibility of perpetual flight.

The proposed power management strategy also sustains the perpetual flight capabilities of the solar-powered UAV. However, the results in Sections 4.1 and 4.2 do not take into consideration the change in atmosphere condition. Here, the crucial parameters which affect the system include the temperature, especially on the conversion efficiency of solar energy and the density of air, which relates to the flow of air around the aircraft. This assumption made in the simulation results intends to make similar comparison with existing trajectory models as discussed in Sections 4.1 and 4.2. 


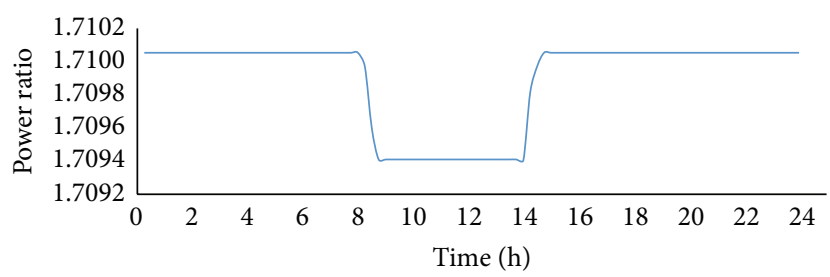

FIgURE 3: Trend of the power ratio at the initialization of the cruise phase.

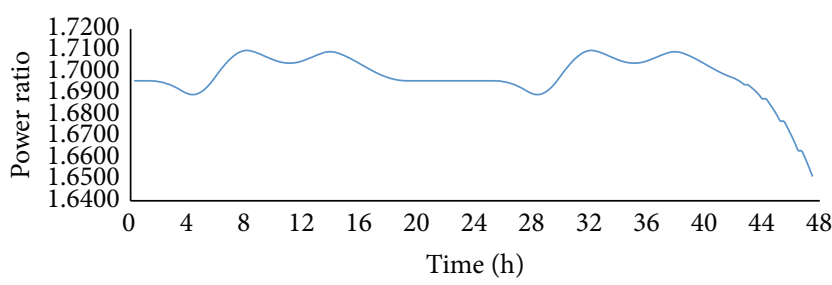

Figure 4: Trend of the power ratio at the initialization of the ascent phase.

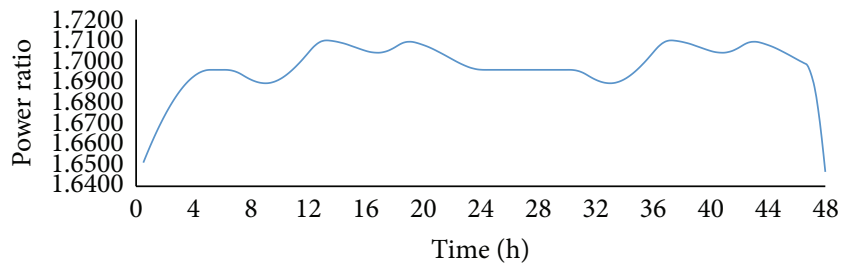

FIGURE 5: Trend of the power ratio at the initialization of the descent phase.

4.1. 24-Hour Mission Simulation. The trend of the power ratio with the start hours of the cruise phase is illustrated in Figure 3. The plotted data indicate that a high power ratio is obtained from the 0.25 th hour until the 8.5th hour with the highest power ratio at approximately 1.71 . This power ratio is achieved again from the 14 th to the 24 th hour. Hence, this time region of the optimal power ratio is the best for a cruise flight. This finding also emphasizes that the region of the 9th to 13 th hour is unfavorable for a cruise flight. Figure 4 presents the power ratio achieved when the UAV begins to ascend. The power ratio fluctuates. However, four peaks of the net power ratio emerge, with an approximate value of 1.71 if the ascent begins at the 8 th, 14th, 32nd, and 37th hours of flight.

The trend of the power ratio at the initialization of the descent phase is shown in Figure 5. Similar to that in the ascent phase, the power ratio at the beginning of the descent phase fluctuates over time. In this instance, the highest net power ratio obtained is approximately 1.71 at the four peaks, during the 13th, 19th, 37th, and 43rd hours of the descent phase. The simulation results are also plotted in $3 \mathrm{D}$ to analyze the trend line of the power ratio for a few phase combinations. These plots help determine the suitable combination of start times for the phases to obtain the optimum power ratio. For instance, Figure 6 illustrates the combination of start times for the cruise and descent phases and the corresponding power ratios that can be achieved.

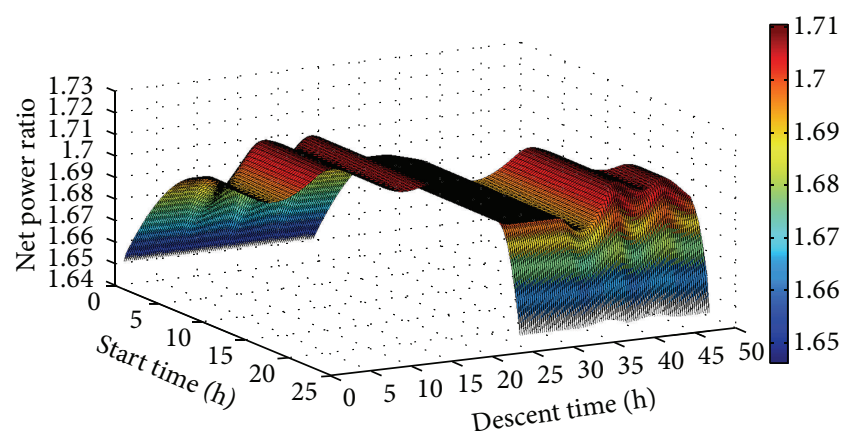

FIgURE 6: Trend of the power ratio with respect to the start time of the cruise and descent phases.

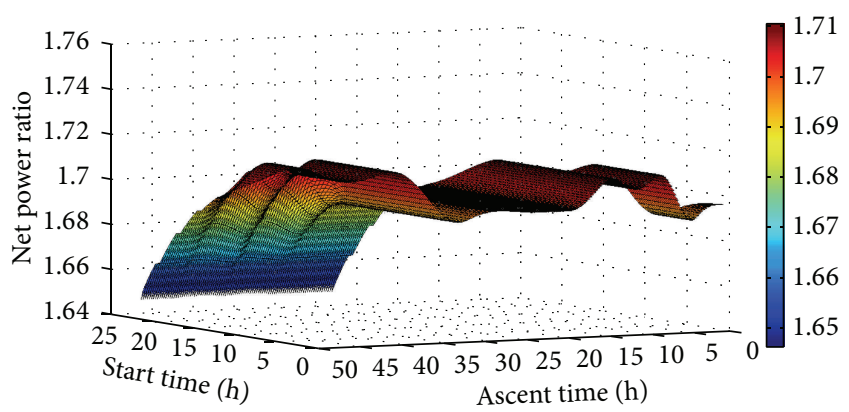

FIGURE 7: Trend of the power ratio with respect to the start times of the cruise and ascent phases.

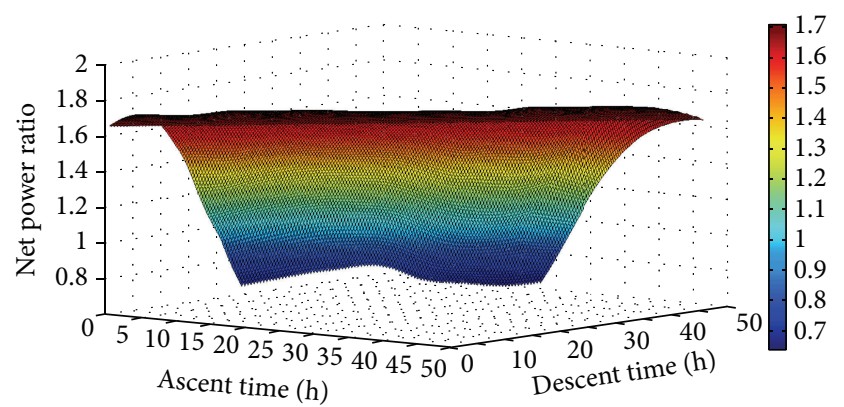

FIGURE 8: Trend of the power ratio with respect to the start times of the ascent and descent phases.

Figure 7 shows the combination of the start times for the cruise and ascent phases to determine the optimum power ratio. The trend line of the power ratio for the combination of the start times of the cruise and descent phases and cruise and ascent phases fluctuates throughout the time of flight. However, for the combination of the start times of the ascent and descent phases shown in Figure 8, the maximum achievable power ratio is quite constant at approximately 1.71 . Thus, the start times of the ascent and descent phases do not significantly affect the power ratio compared with the start time of the cruise phase.

Based on these simulation results, the optimum region for UAV to cruise is from the 0.25 th hour to the 8.5 th hour and from the 14th hour to the 24th hour. The best point for the 


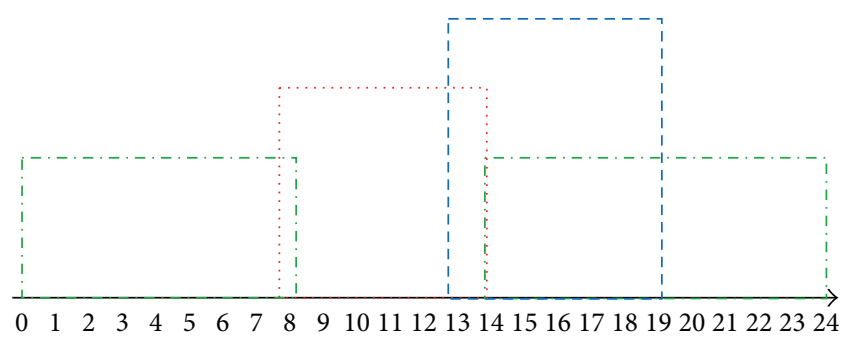

(h)

-.. Cruise

...... Ascend

- - - Descend

FIGURE 9: Recommended mission profile phase region.

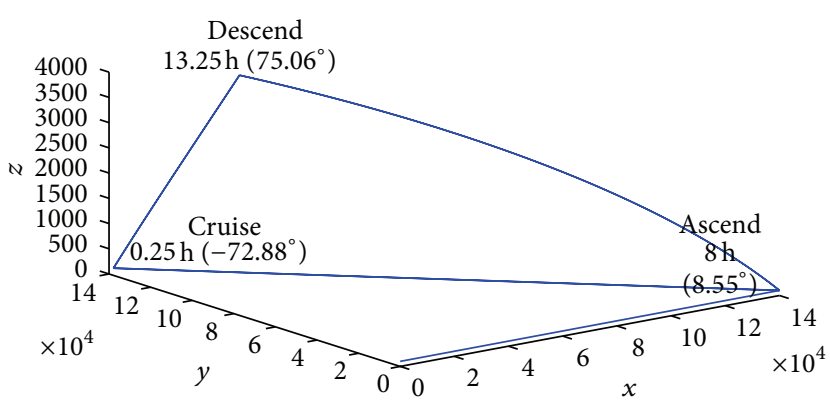

FIGURE 10: Recommended mission trajectory.

UAV to ascend is at the interval of the 8th hour to the 14th hour. The optimum region for the descent phase is the region of the 13th hour to the 19th hour. The region of the optimal mission profile phases over time is illustrated in Figure 9.

The recommended mission profile trajectory obtained based on the extensive simulation is plotted in Figure 10. Here, the cruise phase of the UAV begins at the 0.25th hour, when the solar elevation angle is $-72.88^{\circ}$. The UAV continues to cruise until the 8th hour, when the solar elevation angle is $8.55^{\circ}$. At this point, the UAV is recommended to begin its ascent flight with a minimum climb rate to gain gravitational potential energy. At the 13.25th hour, when the solar elevation angle is $75.06^{\circ}$, the UAV reaches the optimal time to descend without consuming any power. The descent phase ends when the UAV reaches its initial altitude.

A comparison of the SOC for the proposed model and a previous model [11] for a simulated 24-hour flight mission using the same UAV model (Table 1) is presented in Figure 11. The minimum SOC is achieved using the proposed model with a value of $0.6308 \mathrm{C}$, which is slightly lower than the original value by $0.32 \%$. This result indicates that the proposed model exhibits better battery utilization than the original model. Moreover, the recommended lower SOC limit for a rechargeable lithium polymer battery is $0.25 \mathrm{C}[1,7]$.

A detailed comparison of the parameters of the proposed and previous models is given in Table 2. The proposed model improves the net power ratio by $30.84 \%$. This value can reach 31.04 megawatts for UAV operations over a year. By adopting

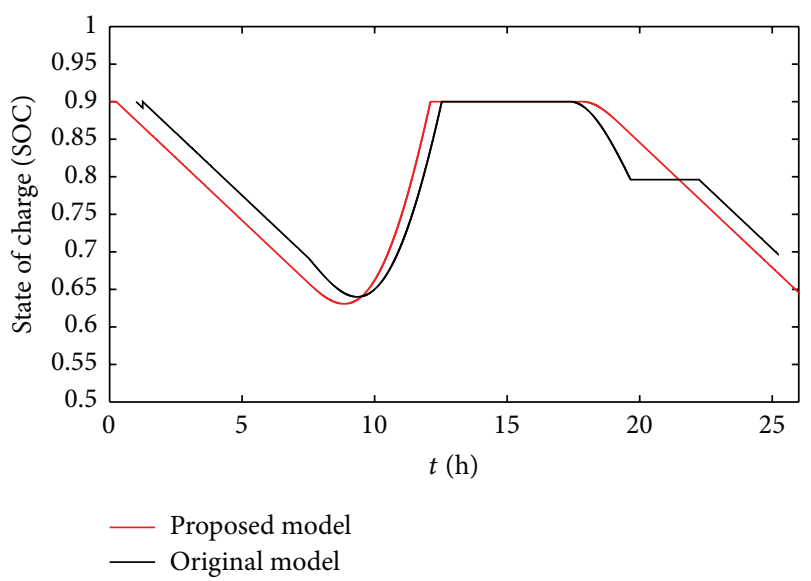

FIGURE 11: Battery SOC for a 24-hour flight.

TABLE 2: 24-hour flight mission performance data.

\begin{tabular}{lccc}
\hline Parameters & Original model & $\begin{array}{c}\text { Proposed } \\
\text { model }\end{array}$ & $\begin{array}{c}\text { Improvement } \\
(\%)\end{array}$ \\
\hline $\begin{array}{l}\text { Power ratio } \\
\text { Ceiling altitude } \\
(\mathrm{m})\end{array}$ & 1.3070 & 1.7101 & 30.84 \\
\hline
\end{tabular}

TABLE 3: Comparison of the proposed model with different approaches.

\begin{tabular}{lcc}
\hline Approaches & Power ratio & $\begin{array}{c}\text { Improvement } \\
(\%)\end{array}$ \\
\hline $\begin{array}{l}\text { Flight performance parameters } \\
{[3]}\end{array}$ & 1.0300 & 66.03 \\
$\begin{array}{l}\text { Aircraft parameters optimization } \\
{[12]}\end{array}$ & 1.1125 & 53.71 \\
$\begin{array}{l}\text { Energy management system [9] } \\
\text { Proposed power management } \\
\text { strategy }\end{array}$ & 1.3070 & 30.84 \\
\hline
\end{tabular}

the proposed power management strategy, UAV operations at a lower altitude may avoid the highly volatile wind region.

A comparison of the power ratio performance of the proposed power management strategy and that of various approaches $[3,9,12]$ is listed in Table 3 . The concept of the proposed strategy significantly improves the power ratio by at least $30.84 \%$ relative to the other approaches for perpetual solar-powered UAV missions. Hence, the proposed power management strategy may be a potential alternative for solarpowered UAV operations.

4.2. 72-Hour Perpetual Mission Simulation. A case study for a perpetual flight mission was simulated using the proposed power management strategy. This step is crucial for determining whether or not the battery pack contains sufficient SOC continuously for a sustainable powered flight. Thus, a simulation is conducted for a 72-hour mission to validate whether or not the minimal battery SOC stays within the preset boundary, as shown in Figure 12. 


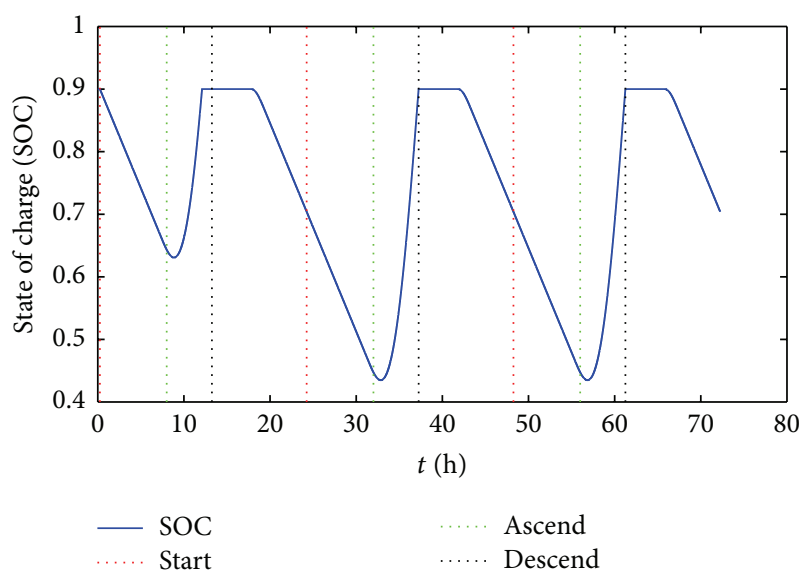

Figure 12: Battery SOC for 72 hours.

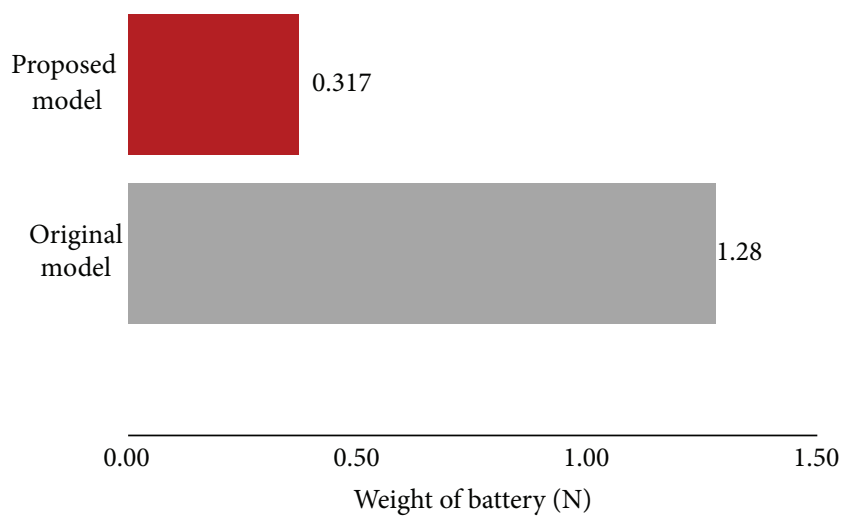

FIGURE 13: Battery weight comparison.

The simulation of a 72-hour perpetual mission achieved a power ratio of 1.7100 , which is slightly lower than that of a 24-hour flight mission. However, this difference is almost negligible. The SOC battery level maintained a consistent cycle for more than 48 hours during the first day at a fully charged state. This result further reaffirms the possibility of operating self-sustainable solar-powered UAV missions for months and even years.

In addition, the minimal battery SOC level remained effectively within the boundary, with a value of $0.4348 \mathrm{C}$. As mentioned earlier, a fully utilized battery is recommended to be $0.25-0.90 \mathrm{C}$. Thus, in this case study, battery weight was significantly reduced from $1.28 \mathrm{~N}$ to $0.317 \mathrm{~N}$ as shown in Figure 13. Here, $s$ total of $75.23 \%$ of the battery's weight was reduced. Since UAV battery weight is roughly $30 \%$ of the overall take-off weight of UAV, the overall weight of the UAV can be reduced by $22.57 \%$. This finding further affirms the potential of the new model to achieve perpetual flight.

\section{Conclusion}

In conclusion, this study proposes adopting vertical cylindrical trajectory with an additional element by defining the optimal time of mission transition in relation to the elevation angle of the sun, which improves the overall gained power ratio. Structuring the mission phases to define the start-endtime of the ascent, cruise, and descent phases of a flight increases the saved energy and improves aircraft endurance. This approach improves the net power ratio by $30.84 \%$ compared with other techniques. As a result, the battery weight may be massively reduced by $75.23 \%$. In addition, the proposed strategy also enables a lower-altitude flight, which protects the UAV from highly volatile winds in high-altitude regions. Therefore, the proposed power management strategy confirms that perpetual solar-powered UAV operations are feasible and sustainable.

\section{Nomenclature}

\begin{tabular}{|c|c|}
\hline$r:$ & Radius \\
\hline$x(t):$ & Displacement in $x$-axis \\
\hline$y(t)$ & Displacement in $y$-axis \\
\hline$z(t):$ & Displacement in $z$-axis \\
\hline$\dot{z}(t)$ & Velocity in $z$-axis \\
\hline$\ddot{z}(t):$ & Acceleration in $z$-axis \\
\hline$\varphi(t):$ & Heading angle \\
\hline$\dot{\varphi}(t)$ & Heading angle rate \\
\hline$\ddot{\varphi}(t):$ & Heading angle acceleration \\
\hline$V_{\text {Power }_{\text {min }}}:$ & Minimal power velocity \\
\hline$V_{\text {sink }_{\text {min }}}:$ & Minimum sink rate \\
\hline ceiling: & Ceiling altitude \\
\hline$m:$ & Weight of UAV \\
\hline$\rho_{\infty}:$ & Air density \\
\hline$S:$ & Wing's surface area \\
\hline$\alpha:$ & Angle of attack \\
\hline$g:$ & Gravitational acceleration \\
\hline se: & Solar elevation angle \\
\hline az: & Solar azimuth angle \\
\hline$i:$ & Solar inclination angle \\
\hline$m_{\text {bat }}:$ & Mass of battery \\
\hline$Q:$ & Battery capacity \\
\hline$R:$ & Battery resistance \\
\hline$V_{\mathrm{oc}}:$ & Battery open circuit voltage \\
\hline SOC: & State of charge of battery \\
\hline SÖC: & Rate of change of SOC \\
\hline$\eta_{E_{b}}:$ & Efficiency of battery pack \\
\hline$\eta_{\text {prop }}:$ & Efficiency of propeller \\
\hline$\eta_{\text {sol }}:$ & Efficiency of solar panel \\
\hline UAV: & Unmanned aerial vehicle \\
\hline$V:$ & Magnitude of velocity \\
\hline$V_{t}(t):$ & Tangential velocity \\
\hline$V_{n}(t):$ & Normal velocity \\
\hline$V_{z}(t):$ & Ascending velocity \\
\hline$a:$ & Acceleration \\
\hline$a_{t}(t):$ & Tangential acceleration \\
\hline$a_{n}(t)$ & Normal acceleration \\
\hline$a_{z}(t):$ & Ascending acceleration \\
\hline$\gamma(t)$ & Flight path angle \\
\hline e: & Oswald efficiency factor \\
\hline AR: & Aspect ratio \\
\hline$C_{D_{0}}:$ & Zero-lift drag coefficient \\
\hline
\end{tabular}


$C_{l_{0}}: \quad$ Zero angle of attack lift coefficient

$C_{l_{\alpha}}: \quad$ Lift curve slope

$C_{L}: \quad$ Lift coefficient

$C_{D}$ : Drag coefficient

$D$ : Drag

L: $\quad$ Magnitude of lift

$L_{t}: \quad$ Tangential lift

$L_{n}: \quad$ Normal lift

$L_{z}:$ Vertical lift

T: $\quad$ Thrust required

Psd: Solar spectral density

$P_{\text {out }}$ : Power consumption

$P_{\text {in }}$ : Power derived from sun

$P_{\text {bat }}$ : Power of battery

$E_{\text {out }}:$ Energy consumption

$E_{\text {in }}$ : Energy derived from sun

$K$ : Aerodynamic coefficient

EMS: Energy management strategy.

\section{Competing Interests}

The authors declare that they have no competing interests.

\section{Acknowledgments}

This study was supported by the Universiti Sains Malaysia (Grant no. 304/PAERO/60312047).

\section{References}

[1] P. Rajendran and H. Smith, "The development of a small solar powered electric unmanned aerial vehicle systems," in 4th Mechanical and Manufacturing Engineering, vol. 465-466, pp. 345-351, 2014.

[2] H. Saghar and M. Mehran, "Energy aware aerial surveillance for a long endurance solar-powered UAV," in Proceedings of the AIAA Guidance, Navigation, and Control (GNC) Conference, American Institute of Aeronautics and Astronautics, Boston, Mass, USA, 2013.

[3] X.-Z. Gao, Z.-X. Hou, Z. Guo, R.-F. Fan, and X.-Q. Chen, “The equivalence of gravitational potential and rechargeable battery for high-altitude long-endurance solar-powered aircraft on energy storage," Energy Conversion and Management, vol. 76, pp. 986-995, 2013.

[4] P. Rajendran and H. Smith, "Future trend analysis on the design and performance of solar-powered electric unmanned aerial vehicles," Advanced Materials Research, vol. 1125, pp. 635-640, 2015.

[5] P. Rajendran and H. Smith, "Implications of longitude and latitude on the size of solar-powered UAV," Energy Conversion and Management, vol. 98, pp. 107-114, 2015.

[6] P. Rajendran, H. Smith, and M. Hazim Bin Masral, "Modeling and simulation of solar irradiance and daylight duration fora high-power-output solar module system," Applied Mechanics and Materials, vol. 629, pp. 475-480, 2014.

[7] P. Rajendran and H. Smith, "Review of solar and battery power system development for solar-powered electric unmanned aerial vehicles," Advanced Materials Research, vol. 1125, pp. 641647, 2015.
[8] H. Smith and P. Rajendran, "Review of the elementary aspect of small solar-powered electric unmanned aerial vehicles," Australian Journal of Basic and Applied Sciences, vol. 8, no. 15, pp. 252-259, 2014.

[9] X.-Z. Gao, Z.-X. Hou, Z. Guo, P. Wang, and J.-T. Zhang, "Research on characteristics of gravitational gliding for highaltitude solar-powered unmanned aerial vehicles," Proceedings of the Institution of Mechanical Engineers, Part G, vol. 227, no. 12, pp. 1911-1923, 2013.

[10] A. T. Klesh and P. T. Kabamba, "Solar-powered aircraft: energyoptimal path planning and perpetual endurance," Journal of Guidance, Control, and Dynamics, vol. 32, no. 4, pp. 1320-1329, 2009.

[11] S. Hosseini, R. Dai, and M. Mesbahi, "Optimal path planning and power allocation for a long endurance solar-powered UAV," in Proceedings of the American Control Conference (ACC '13), Washington, DC, USA, June 2013.

[12] S. C. Spangelo, E. G. Gilberty, A. T. Klesh, P. T. Kabamba, and A. R. Girard, "Periodic energy-optimal path planning for solarpowered aircraft," in Proceedings of the AIAA Guidance, Navigation, and Control Conference and Exhibit, Chicago, Ill, USA, August 2009.

[13] X.-Z. Gao, Z.-X. Hou, Z. Guo, J.-X. Liu, and X.-Q. Chen, "Energy management strategy for solar-powered high-altitude long-endurance aircraft," Energy Conversion and Management, vol. 70, pp. 20-30, 2013.

[14] X.-Z. Gao, Z.-X. Hou, Z. Guo, X.-Q. Chen, and X.-Q. Chen, "Joint optimization of battery mass and flight trajectory for high-altitude solar-powered aircraft," Proceedings of the Institution of Mechanical Engineers, Part G: Journal of Aerospace Engineering, vol. 228, no. 13, pp. 2439-2451, 2014.

[15] R. Dai, "Path planning of solar-powered unmanned aerial vehicles at low altitude," in Proceedings of the IEEE 56th International Midwest Symposium on Circuits and Systems (MWSCAS '13), pp. 693-696, Columbus, Ohio, USA, August 2013.

[16] A. T. Klesh and P. T. Kabamba, "Energy-optimal path planning for Solar-powered aircraft in level flight," in Proceedings of the AIAA-2007-6655, AIAA Guidance, Navigation and Control Conference and Exhibit, Hilton Head, SC, USA, 2007.

[17] E. E. Pinar, Energy optimal path planning of an unmanned solar powered aircraft [M.S. thesis], The Graduate School of Natural and Applied Sciences, Middle East Technical University, Ankara, Turkey, 2013.

[18] J. Anderson, Aircraft Performance and Design, McGraw-Hill, 1999.

[19] G. A. Landis, A. Colozza, and C. M. LaMarre, "Atmospheric flight on venus: a conceptual design," Journal of Spacecraft and Rockets, vol. 40, no. 5, pp. 672-677, 2003.

[20] G. Sachs, J. Lenz, and F. Holzapfel, "Periodic optimal flight of solar aircraft with unlimited endurance performance," Applied Mathematical Sciences, vol. 4, no. 73-76, pp. 3761-3778, 2010.

[21] R. Bencatel, J. Tasso De Sousa, and A. Girard, "Atmospheric flow field models applicable for aircraft endurance extension," Progress in Aerospace Sciences, vol. 61, pp. 1-25, 2013.

[22] V. Badescu and P. T. Landsberg, "Statistical thermodynamic foundation for photovoltaic and photothermal conversion. I. Theory," Journal of Applied Physics, vol. 78, no. 4, pp. 2782-2792, 1995.

[23] V. Badescu and P. T. Landsberg, "Statistical thermodynamics foundation for photovoltaic and photothermal conversion. II. Application to photovoltaic conversion," Journal of Applied Physics, vol. 78, no. 4, pp. 2793-2802, 1995. 
[24] P. T. Landsberg and V. Badescu, "Carnot factor in solar cell efficiencies," Journal of Physics D: Applied Physics, vol. 33, no. 22, pp. 3004-3008, 2000.

[25] V. Badescu, "Spectrally and angularly selective photothermal and photovoltaic converters under one-sun illumination," Journal of Physics D: Applied Physics, vol. 38, no. 13, pp. 2166-2172, 2005.

[26] A. S. Oshaba, E. S. Ali, and S. M. Abd Elazim, "MPPT control design of PV system supplied SRM using BAT search algorithm," Sustainable Energy, Grids and Networks, vol. 2, pp. 51-60, 2015.

[27] A. S. Oshaba and E. S. Ali, "Bacteria foraging: a new technique for speed control of DC series motor supplied by photovoltaic system," WSEAS Transactions on Power Systems, vol. 9, pp. 185195, 2014.

[28] A. S. Oshaba, E. S. Ali, and S. M. Abd Elazim, "ACO based speed control of SRM fed by photovoltaic system," International Journal of Electrical Power and Energy Systems, vol. 67, pp. 529536, 2015. 


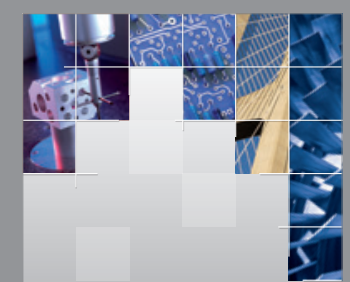

\section{Enfincering}
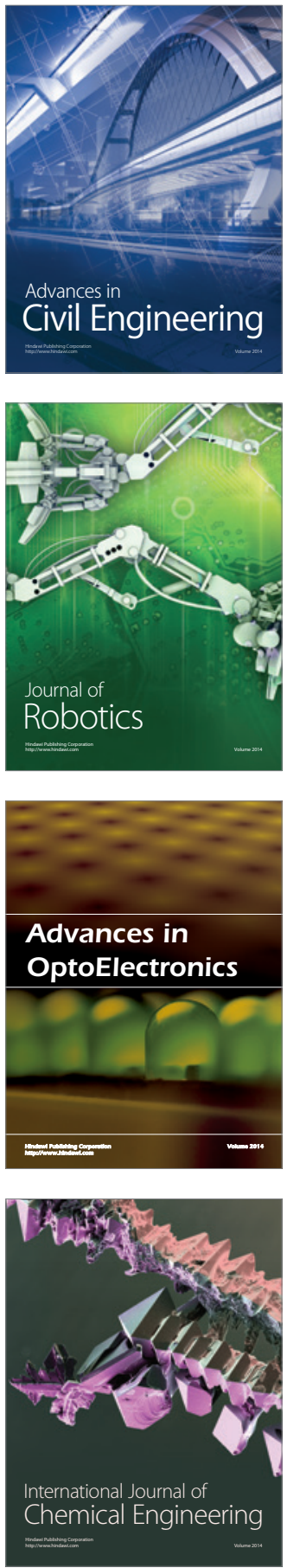

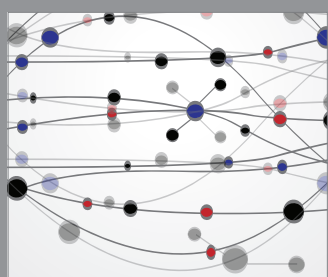

The Scientific World Journal

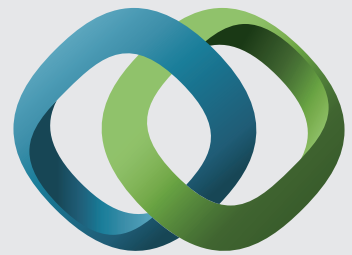

\section{Hindawi}

Submit your manuscripts at

http://www.hindawi.com
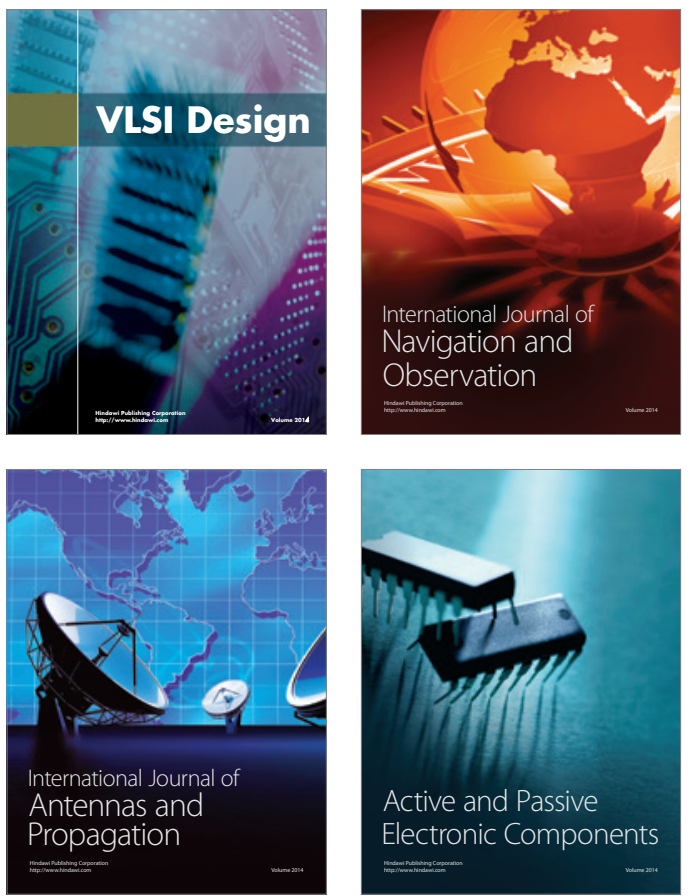
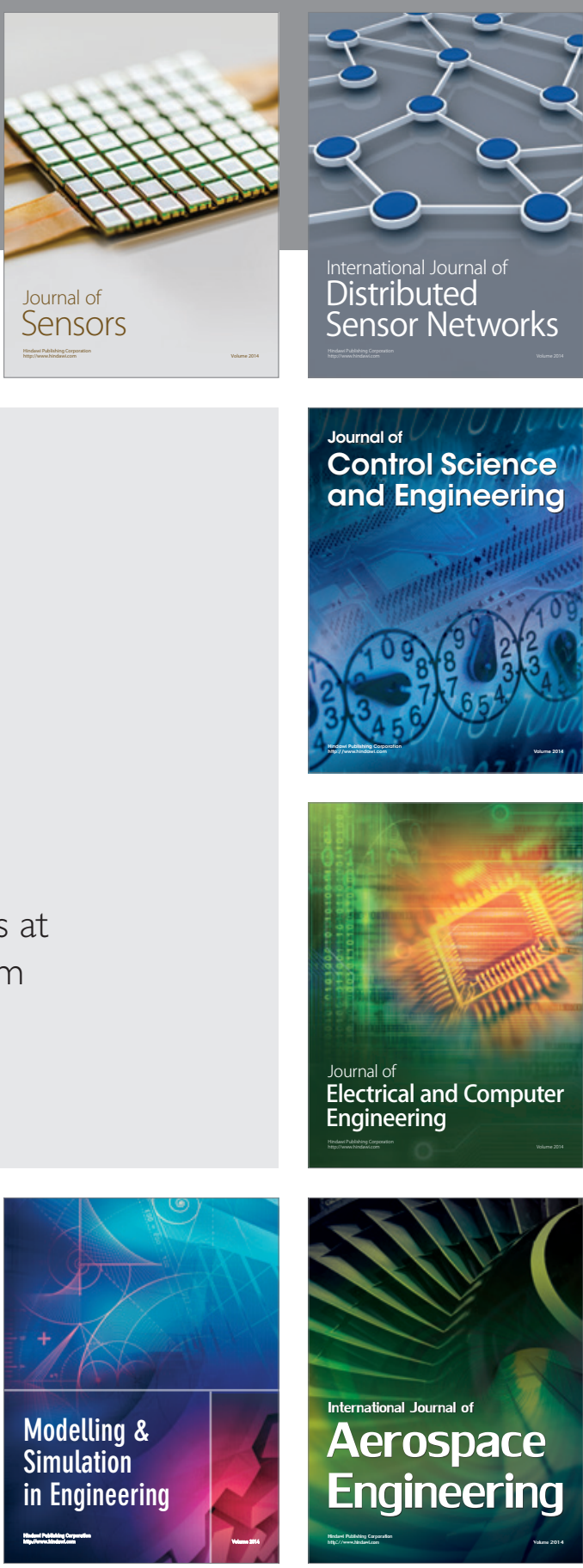

International Journal of

Distributed

Sensor Networks

Journal of

Control Science

and Engineering
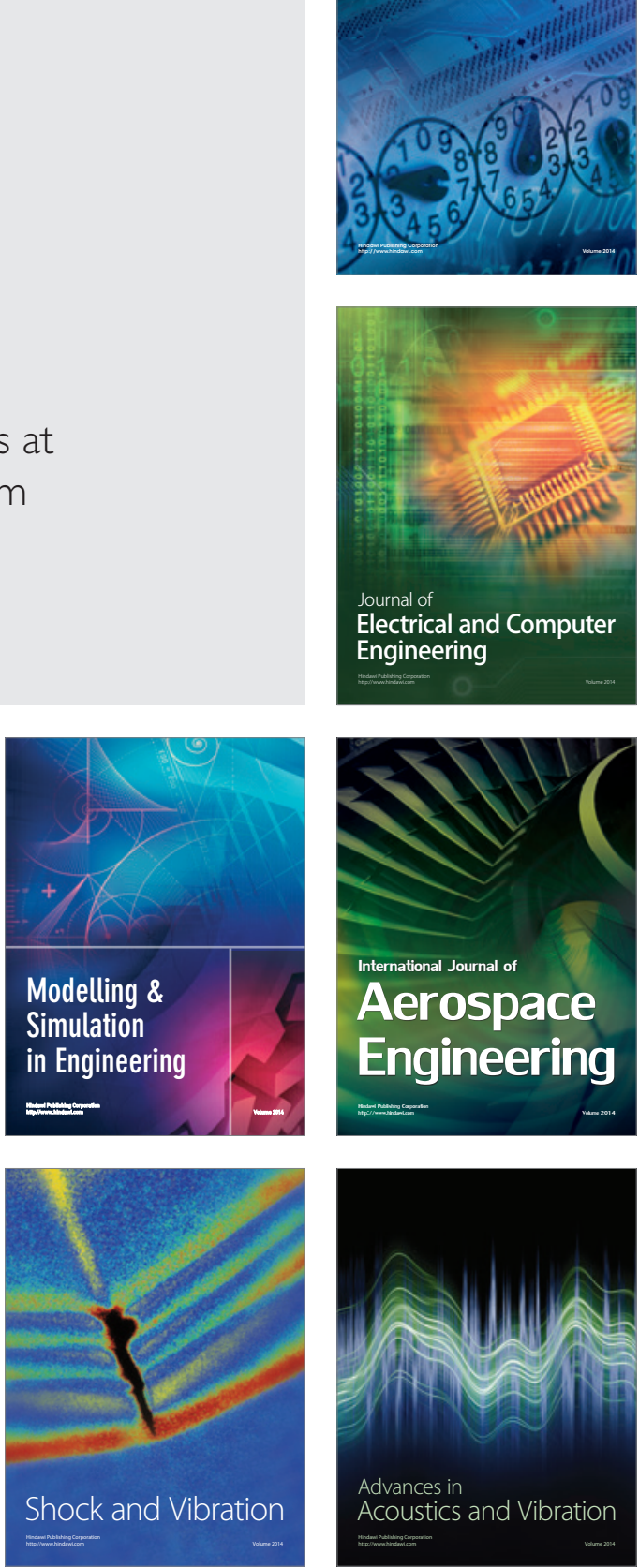